\title{
Seeing is believing: glycosylation in the crystal structure of human myeloperoxidase
}

\author{
L. Krawczyk ${ }^{1}$, S. Semwal ${ }^{1}$, G. Roos ${ }^{1}$, P. Van Antwerpen², J. Bouckaert ${ }^{1}$ \\ ${ }^{1}$ Unité de Glycobiologie Structurale et Fonctionnelle, UMR 8576 du CNRS and University of Lille, 50 Avenue de Halley, 59650 \\ Villeneuve d'Ascq, France, \\ ${ }^{2}$ Laboratory of Pharmaceutical Chemistry and Analytical Platform, Faculty of Pharmacy, Université libre de Bruxelles, CP205/05, \\ Boulevard du Triomphe, 1050 Brussels, Belgium. \\ julie.bouckaert@univ-lille.org
}

Human myeloperoxidase (MPO) was first isolated in 1941 from purulent pleuritis fluid from tuberculosis patients. When neutrophilic polymorphonuclear leukocytes (neutrophils) entrap microbial or other invasive particulates, they release MPO during degranulation. In a respiratory burst of highly reactive oxygen species, MPO catalyzes the production of hypohalous acids, primarily hypochlorous acid in physiologic situations, from hydrogen peroxide. Mammal MPO crystal structures were progressively acquired and encoded in PDB with partial glycosylation identification. Actually, the $N$-glycan composition of native MPO had been thoroughly investigated with mass spectrometry and shows $5 \mathrm{~N}$-glycans at positions 323, 355, 391, 483 and 729 [1]. MPO's enzymatic activity was shown to be modulated by hyper-truncation of 2 out of $5 \mathrm{~N}$-glycosylation sites [2].

In our obtained crystal structure at $2.6 \AA$ resolution containing 4 disulfide-linked homodimers of MPO (Fig. 1), an interesting collection of glycans have been characterized using the iterative process of crystallographic refinement and model building. We compared those with the glycans from proteomics studies and from 18 human MPO structures in the PDB. We made use of the Symbol Nomenclature for Glycans (SNFG) to illustrate congruence in the experimental data. In conclusion, we found each of the 5 glycosylation sites either non-glycosylated or glycosylated with hyper-truncated paucimannosidic, high-mannose and complex $N$ glycans, with the $N$-acetyl- $\beta$-D-glucosamine (GlcNAc) core-type asparagine-linked glycans on Asn355 or Asn391 sites [2] gatekeeping the funnel towards the ROS-activated heme group. Our results perfectly illustrate the power of protein crystallography to resolve protein glycosylation.
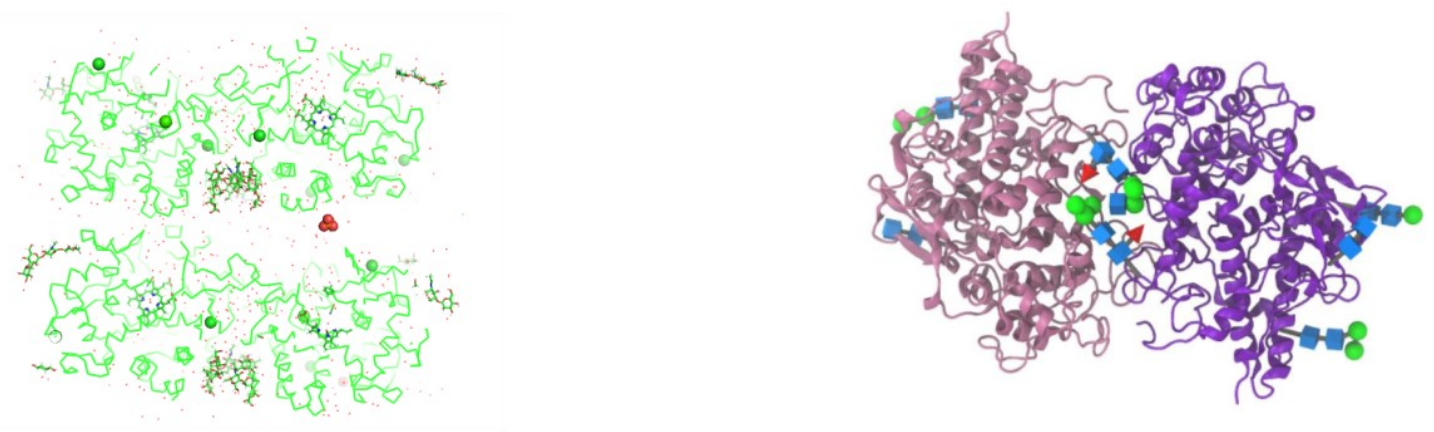

Figure 1. (a) Glycosylation as part of protein crystal structures (2 MPO dimers), (b) a sweet handshake holds the dimer together

[1] Van Antwerpen, P., Slomianny, M. C., et al., (2010) Glycosylation pattern of mature dimeric leukocyte and recombinant monomeric myeloperoxidase: glycosylation is required for optimal enzymatic activity. J Biol Chem 285, 16351-16359. 10.1074/jbc.M109.089748

[2] Tjondro, H. C., Ugonotti, J., et al., (2020) Hyper-truncated Asn355- and Asn391-glycans modulate the activity of neutrophil granule myeloperoxidase. J Biol Chem 10.1074/jbc.M109.089748

Keywords: myeloperoxidase; glycosylation; crystal structure, $\mathrm{N}$-glycans 